\title{
The role of self-reported smell and taste disorders in suspected COVID-19
}

\author{
Athanasia Printza ${ }^{1}\left[\right.$. Jannis Constantinidis ${ }^{1}$
}

Received: 9 May 2020 / Accepted: 18 May 2020 / Published online: 23 May 2020

(c) Springer-Verlag GmbH Germany, part of Springer Nature 2020

\begin{abstract}
Purpose The sudden onset of smell and taste loss has been reported as a symptom related to COVID-19. There is urgent need to provide insight to the pandemic and evaluate anosmia as a potential screening symptom that might contribute to the decision to test suspected cases or guide quarantine instructions.

Methods Systematic review of the PubMed/Medline, Cochrane databases and preprints up to May 3, 2020. Combined search terms included: "COVID-19", "SARS-CoV-2", "coronavirus", "nose", "anosmia", "hyposmia", "olfactory loss", "smell loss", "taste loss", and "hypogeusia".

Results Our search identified 18 reviewed articles and 6 manuscript preprints, including a large epidemiological study, four observational case series, five case-controlled studies, five cross-sectional studies, five case series of anosmic patients and four electronic surveys. Great methodological differences were noted. A significant prevalence of anosmia is reported in COVID-19 patients. Controlled studies indicate that anosmia is more common in COVID-19 patients than in patients suffering from other viral infections or controls. Most of the studies reported either smell loss or smell plus taste loss. Less severe COVID-19 disease is related to a greater prevalence of anosmia. A quick recovery of the smell loss may be expected in most COVID-19 cases.

Conclusion Anosmia is more prevalent in COVID-19 patients than in patients suffering from other respiratory infections or controls.
\end{abstract}

Keywords Anosmia $\cdot$ COVID-19 $\cdot$ Olfactory dysfunction $\cdot$ Loss of smell $\cdot$ SARS-CoV-2 $\cdot$ Taste

Since the outbreak of the COVID-19 pandemic, observations and scientific reports have been accumulating rapidly that sudden anosmia and taste disorders are symptoms associated with the COVID-19 infection [1,2]. Recently the World Health Organization has included the loss of smell or taste as a new symptom of COVID-19 infection as have many Health Authorities after a surge of publications and press releases that pointed to anosmia as a potential screening symptom that might contribute to the decision to test suspected cases or guide quarantine instructions. Subsequently, Otolaryngologic and Rhinologic Societies have worldwide also advised to consider patients with newly acquired sudden loss of smell as potentially positive for SARS-CoV-2. Given

Athanasia Printza

aprintza@auth.gr; nan@med.auth.gr

$1 \quad$ 1st Otolaryngology Department, Medical Faculty, School of Health Sciences, Aristotle University of Thessaloniki, 54124 Thessaloníki, Greece the increase of publications concerning anosmia, and the fact that most current research is under "time-pressure" to provide insight to the pandemic, we undertook a review of the published relevant literature. The objective of this systematic review was to examine existing scientific evidence on the role of anosmia detection in the COVID-19 pandemic and highlight the areas of need for further research.

A computerized search of the PubMed/Medline and Cochrane databases was performed of all indexed studies to identify all relevant manuscripts and preprints up to May 3, 2020. Preprints' repositories included medRxiv. Combined search terms included: "COVID-19", "SARS-CoV-2", "coronavirus", "nose", "anosmia", "hyposmia", "olfactory loss", "smell loss", "taste loss", and "hypogeusia". Adjunctive searches were performed based on the studies that were identified (and their references). Studies were excluded if full texts could not be obtained.

Our search identified 18 reviewed articles published or accepted for publication [1-18] and 6 manuscript preprints 


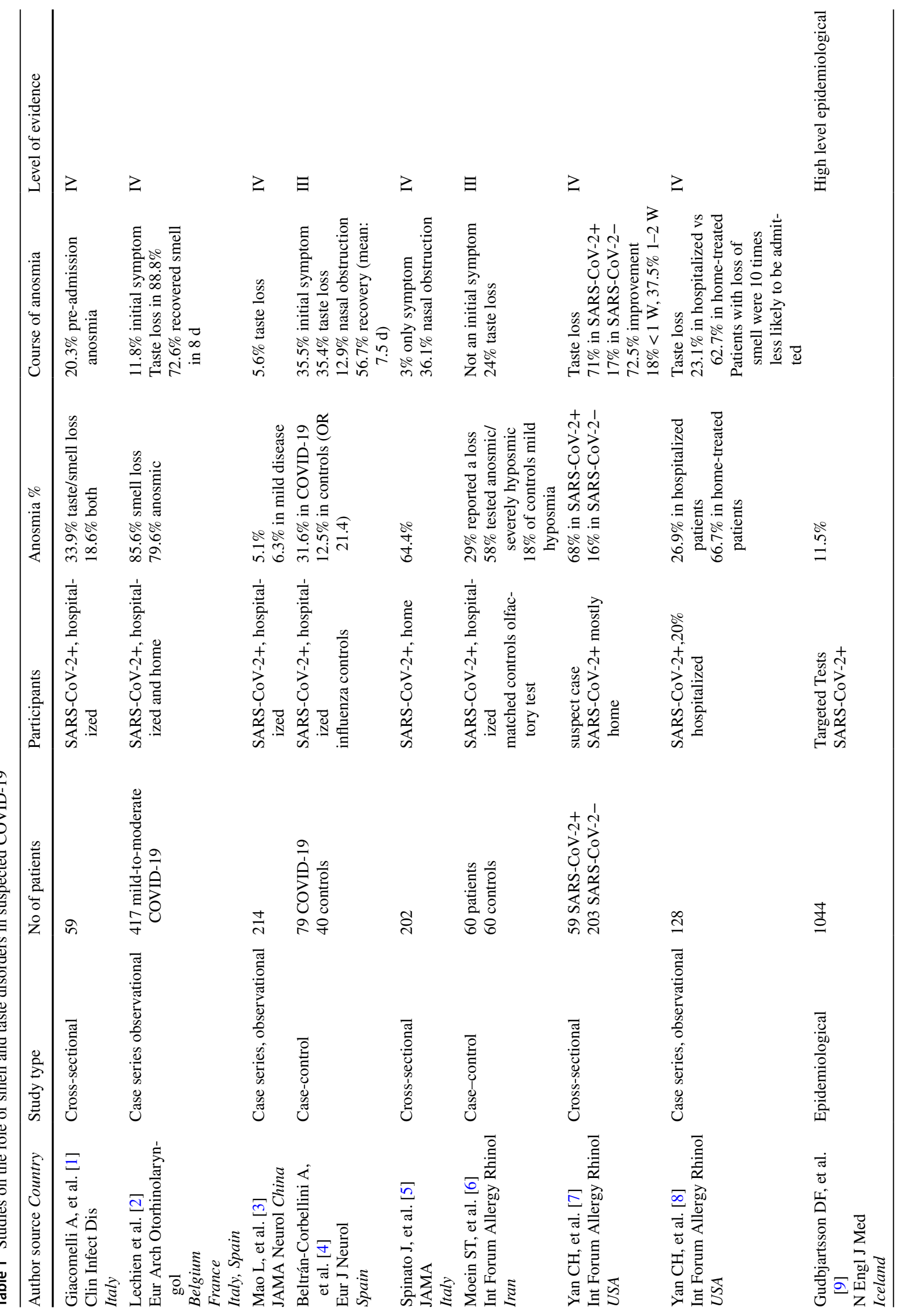




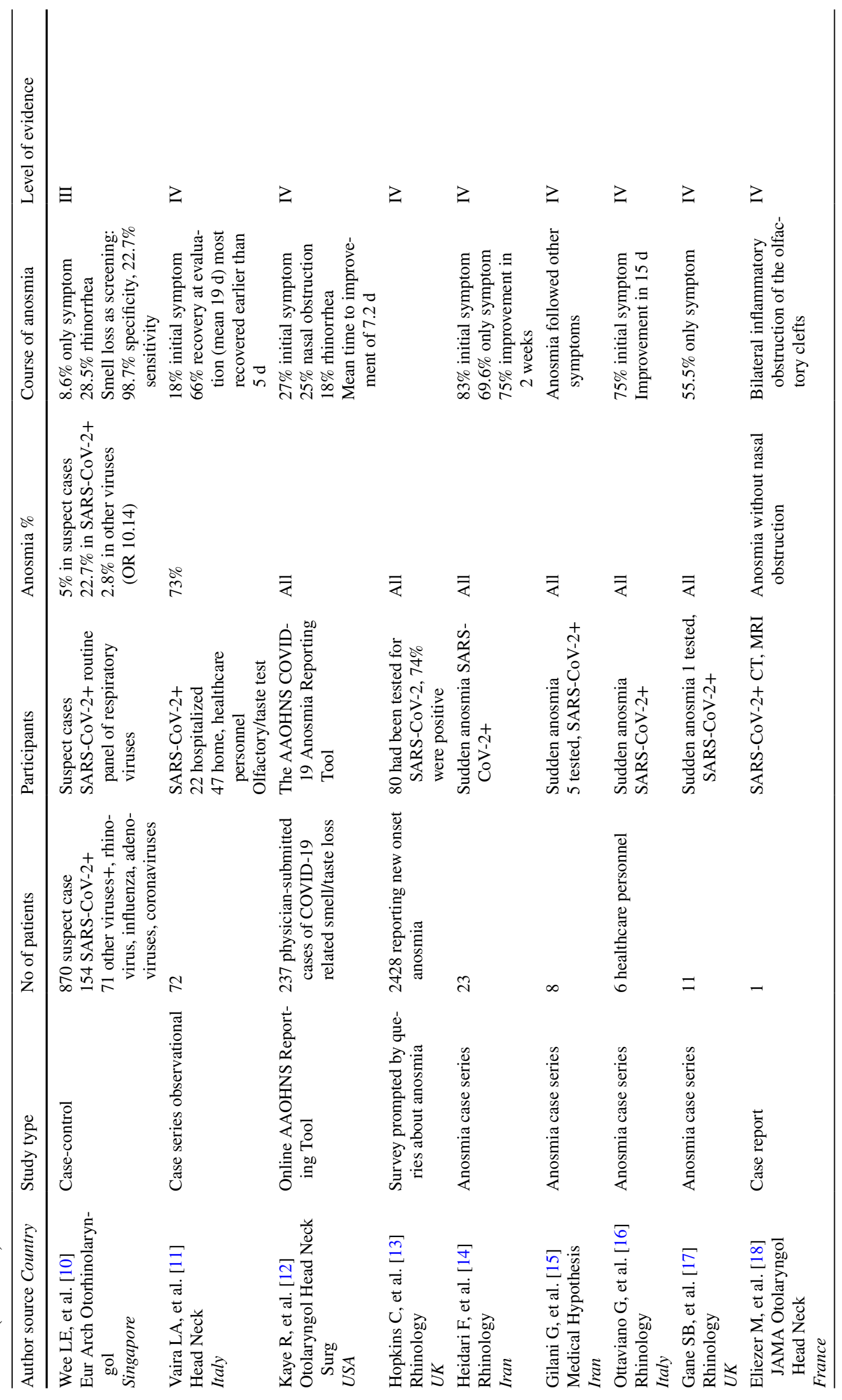




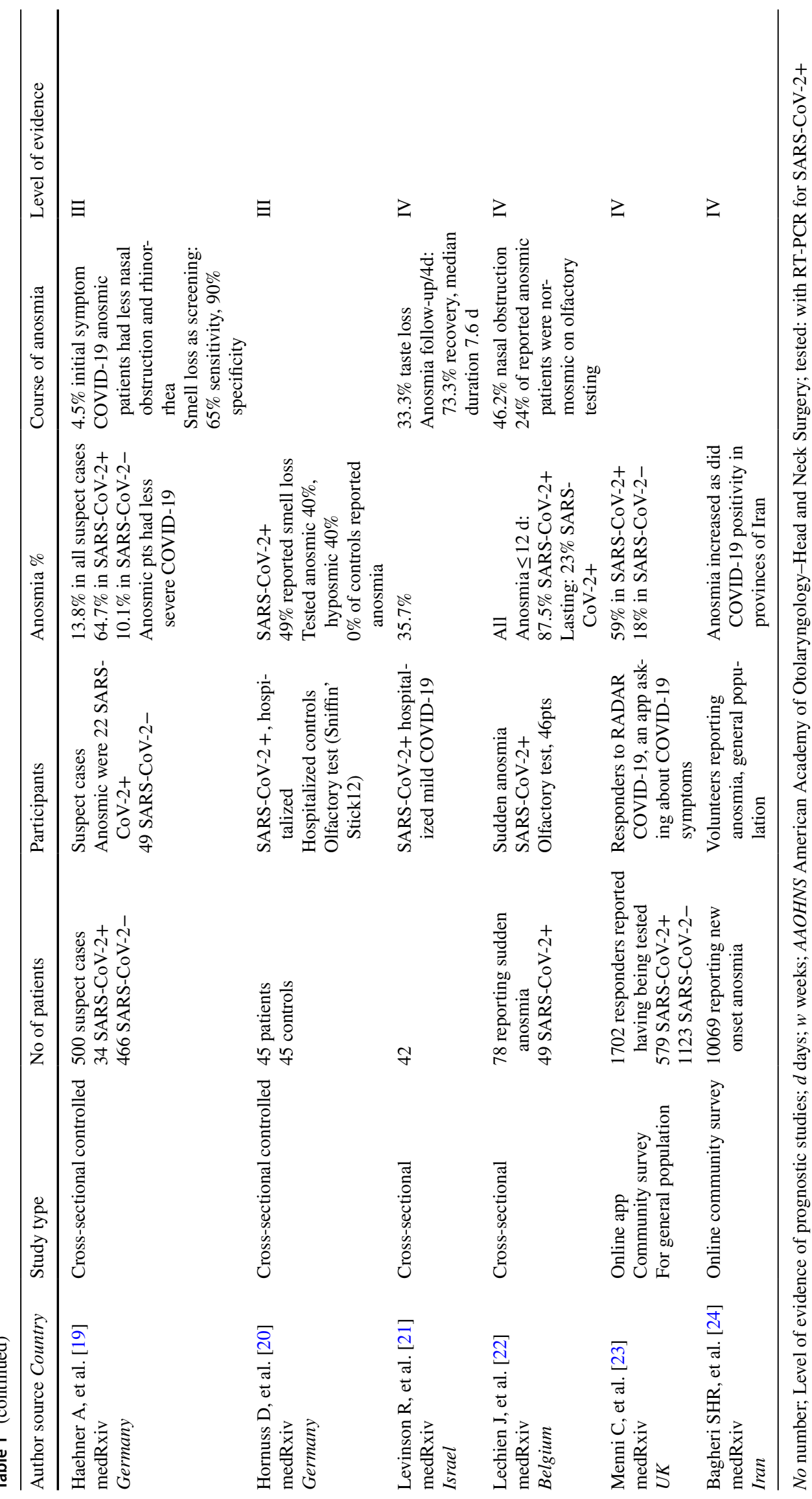


(not peer-reviewed) [19-24] reporting on patients presenting sudden anosmia/taste loss in the context of COVID-19 infection (Table 1). The studies took place from mid-January to mid-April. There were great methodological differences among the studies. A large epidemiological study reported on 9199 persons who were recruited for targeted testing in Iceland [9]. Four large observational case series reported anosmia prevalence in COVID-19 cohorts from China [3], Belgium and other European countries [2], USA [8], Italy [11]. Five case-controlled studies compared the smell/taste loss between SARS-CoV-2 PCR-positive participants and patients PCR-positive for influenza [4], a panel of respiratory viruses [10], matched hospitalized controls [6, 20], and patients tested for influenza-like symptoms for COVID-19 [19]. Five cross-sectional studies of SARS-CoV-2 + patients report on smell/taste loss in patients with varying COVID-19 disease severity $[1,5,7,21,22]$. Four small case series and a case report of anosmic patients, most of whom had been tested for SARS-CoV-2 with various indications [14-18] and four electronic surveys about smell loss [12, 13, 23, 24] commented on the increase of the patients seeking treatment for smell loss during the COVID-19 pandemic. The electronic surveys included the AAOHNS COVID-19 Anosmia Reporting Tool (completed by healthcare providers) [12], The RADAR COVID-19, an online app for the UK general population [23] and two surveys prompted by patients' queries about smell loss (in UK [13] and Iran [24]), one reporting on a small percentage tested for SARS-CoV-2.

Prevalence of smell loss: A large epidemiological study reported a prevalence of $11.5 \%$ for smell loss in 1044 SARSCoV-2 + identified through targeted tests [9]. Four observational case series reported smell loss in their studied cohorts at $5.1 \%$ (hospitalized patients) [3], 26.9\% (hospitalized patients) and $66.7 \%$ (home-treated patients) [8], 73\% (hospitalized and home-treated patients) [11], and 85.6\% (hospitalized and home-treated patients) [2]. The observed discrepancies most likely are related to varying research methods and possible patient selection biases. A significant proportion of the cohort in the studies reporting high anosmia prevalence was healthcare personnel $[2,11]$. Cross-sectional studies found the prevalence of anosmia to be at 33.9\% [1] and $35.7 \%$ [21] for hospitalized patients and 64.4\% [5] and 68\% [7] for out-patients. Smell loss prevalence was compared between COVID-19 patients and control groups in five casecontrolled studies: $22.7 \%$ of COVID-19 patients compared to $2.8 \%$ of patients positive for a panel of respiratory viruses [10], $31.6 \%$ of COVID-19 compared to $12.5 \%$ of influenza [4], $29 \%$ of COVID-19 patients compared to $18 \%$ for controls [6], 49\% compared to $0 \%$ for controls [20], and $64.7 \%$ of COVID-19 patients compared to $10.1 \%$ for respiratory viral illness [19]. The smell loss as a screening symptom was found to show a high specificity and moderate sensitivity for the detection of COVID-19 infection [10, 19].
Clinical characteristics: Most of the studies reported either smell loss or smell plus taste loss. As a presenting symptom, anosmia was rated from 0 to $35 \%$. All the studies that comment on the course of anosmia report quick recovery, in $7-10$ days $[2,4,7,11,14,16,21]$. Nasal obstruction was found at rates ranging from $12.9 \%$ to $46 \%$ and rhinorrhea from $18 \%$ to $28.5 \%$.

While great methodological differences were noted in the reviewed studies, a significant prevalence of anosmia is reported in COVID-19 patients, and controlled studies indicate that anosmia is more common in COVID-19 patients than in patients suffering from other respiratory infections or controls, taking into account seasonal patterns of olfactory dysfunction. Less severe COVID-19 disease is related to a greater prevalence of anosmia. Olfactory testing and treatment decisions should take into account that quick recovery of the smell loss may be expected in most COVID-19 cases.

\section{Compliance with ethical standards}

Conflict of interest The authors have no conflicts of interest.

\section{References}

1. Giacomelli A, Pezzati L, Conti F et al (2020) Self-reported olfactory and taste disorders in SARS-CoV-2 patients: a cross-sectional study. Clin Infect Dis. https://doi.org/10.1093/cid/ciaa330

2. Lechien JR, Chiesa-Estomba CM, De Siati DR et al (2020) Olfactory and gustatory dysfunctions as a clinical presentation of mildto-moderate forms of the coronavirus disease (COVID-19): a multicenter European study. Eur Arch Otorhinolaryngol. https://doi. org/10.1007/s00405-020-05965-1

3. Mao L, Jin H, Wang M et al (2020) Neurologic manifestations of hospitalized patients with coronavirus disease 2019 in Wuhan. China. JAMA Neurol 10:e201127. https://doi.org/10.1001/jaman eurol.2020.1127

4. Beltrán-Corbellini Á, Chico-García JL, Martínez-Poles J et al (2020) Acute-onset smell and taste disorders in the context of Covid-19: a pilot multicenter PCR-based case-control study. Eur J Neurol. https://doi.org/10.1111/ene.14273

5. Spinato G, Fabbris C, Polesel J et al (2020) Alterations in smell or taste in mildly symptomatic outpatients with SARS-CoV-2 infection. JAMA. https://doi.org/10.1001/jama.2020.6771

6. Moein ST, Hashemian SMR, Mansourafshar B et al (2020) Smell dysfunction: a biomarker for COVID-19. Int Forum Allergy Rhinol. https://doi.org/10.1002/alr.22587

7. Yan CH, Faraji M, Prajapati DP, Boone CE (2020) Association of chemosensory dysfunction and Covid-19 in patients presenting with influenza-like symptoms. Int Forum Allergy Rhinol. https:// doi.org/10.1002/alr.22579

8. Yan CH, Faraji F, Prajapati DP, Ostrander BT, DeConde AS (2020) Self-reported olfactory loss associates with outpatient clinical course in Covid-19. Int Forum Allergy Rhinol. https:// doi.org/10.1002/alr.22592 
9. Gudbjartsson DF, Helgason A, Jonsson H et al (2020) Spread of SARS-CoV-2 in the icelandic population. N Engl J Med. https:// doi.org/10.1056/NEJMoa2006100

10. Wee LE, Chan YFZ, Teo NWY et al (2020) The role of selfreported olfactory and gustatory dysfunction as a screening criterion for suspected COVID-19. Eur Arch Otorhinolaryngol. https ://doi.org/10.1007/s00405-020-05999-5

11. Vaira LA, Deiana G, Fois AG et al (2020) Objective evaluation of anosmia and ageusia in COVID-19 patients: single-center experience on 72 cases. Head Neck. https://doi.org/10.1002/hed.26204

12. Kaye R, Chang CWD, Kazahaya K, Brereton J, Denneny JC (2020) COVID-19 anosmia reporting tool: initial findings. Otolaryngol Head Neck Surg. https://doi.org/10.1177/0194599820 922992

13. Hopkins C, Surda P, Kumar N (2020) Presentation of new onset anosmia during the COVID-19 pandemic. Rhinology. https://doi. org/10.4193/rhin20.116

14. Heidari F, Karimi E, Firouzifar M, Khamushian P, Ansari R, Ardehali MM, Heidari F (2020) Anosmia as a prominent symptom of COVID-19 infection. Rhinology 58:302-303. https://doi. org/10.4193/Rhin 20.140

15. Gilani S, Roditi R, Naraghi M (2020) COVID-19 and anosmia in Tehran, Iran. Med Hypotheses 141:109757. https://doi. org/10.1016/j.mehy.2020.109757

16. Ottaviano G, Carecchio M, Scarpa B, Marchese-Ragona R (2020) Olfactory and rhinological evaluations in SARS-CoV-2 patients complaining of olfactory loss. Rhinology. https://doi.org/10.4193/ rhin 20.136

17. Gane SB, Kelly C, Hopkins C (2020) Isolated sudden onset anosmia in COVID-19 infection. A novel syndrome? Rhinology 58:3. https://doi.org/10.4193/rhin20.114

18. Eliezer M, Hautefort C, Hamel AL et al (2020) Sudden and complete olfactory loss function as a possible symptom of COVID-19.
JAMA Otolaryngol Head Neck Surg. https://doi.org/10.1001/ jamaoto.2020.0832

19. Haehner A, Draf J, Draeger S, de With K, Hummel T (2020) Predictive value of sudden olfactory loss in the diagnosis of COVID19. https://doi.org/10.1101/2020.04.27.20081356. medRxiv: 2020.04.27.20081356

20. Hornuss D, Lange B, Schroeter N, Rieg S, Kern WV, Wagner D (2020) Anosmia in COVID-19 patients. https://doi. org/10.1101/2020.04.28.200833. medRxiv: 2020.04.28.20083311

21. Levinson R, Elbaz M, Ben-Ami R et al (2020) Anosmia and dysgeusia in patients with mild SARS-CoV-2 infection. https://doi.org/10.1101/2020.04.11.20055483. medRxiv: 2020.04.11.20055483

22. Lechien J, Cabaraux P, Chiesa-Estomba C et al (2020) Objective olfactory testing in patients presenting with sudden onset olfactory dysfunction as the first manifestation of confirmed COVID-19 infection. https://doi.org/10.1101/2020.04.15.20066 472. medRxiv: 2020.04.15.20066472

23. Menni C, Valdes A, Freydin MB et al (2020) Loss of smell and taste in combination with other symptoms is a strong predictor of COVID-19 Infection. https://doi.org/10.1101/2020.04.05.20048 421. medRxiv: 2020.04.05.20048421

24. Bagheri SHR, Asghari AM, Farhadi M et al (2020) Coincidence of COVID-19 epidemic and olfactory dysfunction outbreak. https://doi.org/10.1101/2020.03.23.20041889. medRxiv: 2020032320041889

Publisher's Note Springer Nature remains neutral with regard to jurisdictional claims in published maps and institutional affiliations. 